gestational age. There is a modest excess of low birthweight and preterm infants in the lower social classes ${ }^{2}$ and a small excess of patients in this series from the higher social classes, but this social class effect is insufficient to account for the findings of this study.

The findings of this study are not consistent with the hypothesis that atopic disease is simply the result of sensitisation in genetically predisposed infants who are immunologically immature. It appears that preterm infants are, if anything, less likely to develop severe atopic eczema than those born at or after term. It is known that preterm infants, if compared with term infants, have a decreased production of specific antibodies to cows' milk proteins during the first six months of life. ${ }^{34}$ Furthermore, the immunological effects of antigens in the neonatal period may be related not only to the state of maturity of the lymphoid system, but also to that of the neonatal digestive system. It has been shown that the absorption of antigenically intact protein in infants less than 33 weeks' gestation may be up to 100 fold greater than at term, ${ }^{5}$ and it is possible that the absorption of large quantities of antigenic material leads to tolerance rather than sensitisation. ${ }^{6}$

There are other possible explanations for these observations, such as protection from preterm delivery in atopic subjects, a lack of atopic disease among twins, or some difference in the method of feeding preterm infants. These possibilities need to be examined in a prospective study to discover the incidence of atopic disease in preterm infants. Protection from severe atopic eczema in preterm infants, if confirmed, could provide important clues about the pathogenesis of atopic disease.

We thank Dr $\mathbf{J}$ Roland for kindly obtaining the control data, and Drs DG Sims, J Couriel, J Golding, HP Roper, and Professor RDH Boyd for their help.

\footnotetext{
References

1 Matthew DJ, Taylor B, Norman AP, Turner MW, Soothill JF. Prevention of eczema. Lancet 1977;i:321-4.

2 Macfarlane A, Mugford M. Birth counts. Statistics of pregnancy and childbirth. London: Her Majesty's Stationery Office, 1984:89-117.

${ }^{3}$ Rieger CHL, Rothberg RM. Development of capacity to produce specific antibody to an ingested food antigen in the premature infant. J Pediatr 1975;87:515-8.

${ }^{4}$ Helms I, Rieger CHL. Decreased production of specific antibodies to cow's milk proteins in premature infants during the first six months of life. Eur J Pediatr 1987;146:131-4.

${ }^{5}$ Roberton DM, Paganelli R, Dinwiddie R, Levinsky RJ. Milk antigen absorption in the preterm and term neonate. Arch Dis Child 1982;57:369-72.

${ }^{6}$ Firer MA, Hosking CS, Hill DJ. Effect of antigen load on development of milk antibodies in infants allergic to milk. Br Med J 1981;283:693-6.
}

Correspondence to Dr TJ David, Booth Hall Children's Hospital, Charlestown Road, Blackley, Manchester M9 2AA.

Accepted 21 December 1987

\title{
Mycotic intracranial abscesses during induction treatment for acute lymphoblastic leukaemia
}

\author{
N K FOREMAN, ${ }^{*}$ M G MOTT, ${ }^{*}$ T M PARKYN, $\dagger$ AND G MOSS*
}

*Paediatric Oncology Unit, Bristol Royal Hospital for Sick Children and †Royal Devon and Exeter Hospital (Wonford), Exeter

SUMMARY A boy with newly diagnosed acute lymphoblastic leukaemia developed mycotic cerebral abcesses despite treatment with amphotericin. $\mathrm{He}$ survived this episode on combination antifungal treatment.

\section{Case report}

A 13 year old boy presented with a week's history of bruising and a white cell count of $19.4 \times 10^{9} / 1$ of which $39 \%$ were blasts. He was febrile at presen- tation, with no obvious site of infection, and was started on intravenous cefuroxime and tobramycin with oral nystatin (100 000 units four times a day). An indwelling Hickman line was inserted and UKALL $\mathrm{X}$ induction, which included prednisolone $40 \mathrm{mg} / \mathrm{m}^{2}$ for 28 days, was commenced.

On the fifth day of treatment he developed glycosuria, with a blood glucose of $25 \mathrm{mmol} / \mathrm{l}$, and required insulin. Antibiotics were discontinued from day five, as bacterial cultures were negative, but were restarted on day 11 as he again became febrile and was neutropenic. The only positive culture was Aspergillus species on a throat swab; chest radio- 
graphy was normal. Intravenous amphotericin was started on day 17 because of persistent fever. On day 28 routine bone marrow confirmed he was in remission. The next day, however, confusional episodes were noted with no accompanying gross neurological signs. Electroencephalography showed an excess of slow waves over the entire cortex.

Psychological testing showed unaffected verbal functioning but he could not cope with non-verbal tasks even at the level of a 5 or 6 year old. He was unable to copy accurately a series of designs. External objects seemed, to him, to change size and shape. Simple judgments made on visuo-spatial information could no longer be made-for example, he could no longer tell the time. In addition, if instructed to perform a series of motor actions, he would repeat the initial action again and again, despite being able to repeat correctly the verbal instructions. Focal brain damage was suspected.

A computed tomogram showed ill defined lucencies posteriorly in both cerebral cortices and anteriorly in the right frontal lobe, with no ring enhancement. The diagnosis was unclear; haemorrhage, infarct, cerebral leukaemia were all considered. Over the next few days he gradually deteriorated and a repeat computed tomogram, performed on

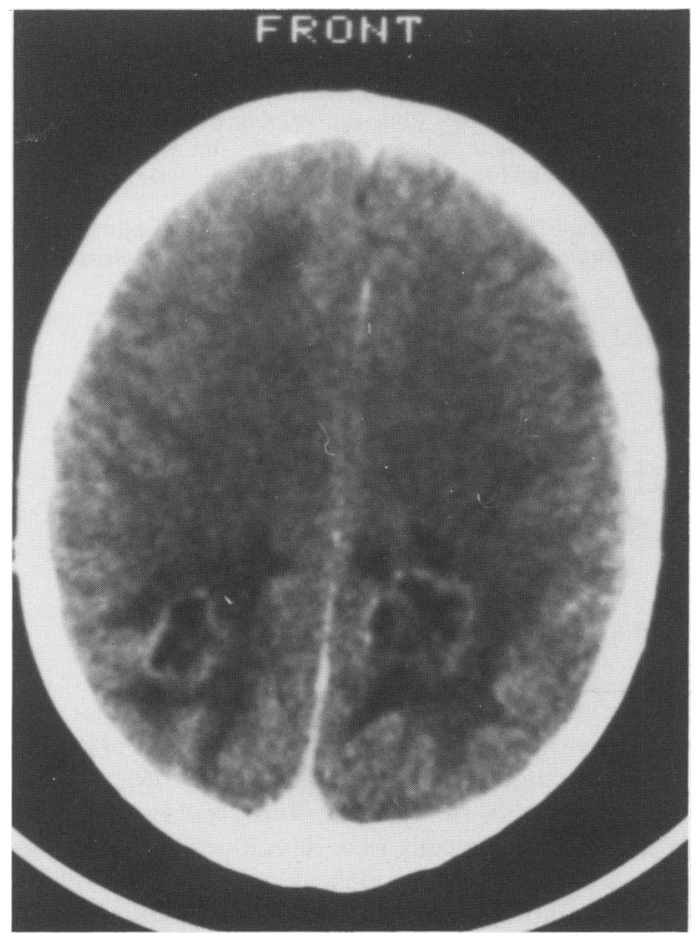

Figure Computed tomogram with intravenous contrast showing the two occipito-parietal lesions. day 37, then showed ring enhancement (figure).

A presumptive diagnosis of mycotic cerebral abscesses was made. No mycotic abscesses were shown in the liver or renal tracts. Blood and urine culture were negative for fungus. Candidial antibody testing, by counter immune electrophoresis, and antigen testing, using Cand-tec (Ramco Laboratories, Houston, Texas), were consistently negative before, during, and after his illness.

Oral flucytosine and oral itraconazole were commenced; the intravenous amphotericin was continued. Stereotactic aspiration of the abscesses was performed guided by computed tomography. Microscopy showed fungal hyphae and culture showed Candida albicans and anaerobic Streptococci. Benzylpenicillin was added, in high dose, to his antibiotic regime. Antifungals were given as follows: amphotericin $1 \mathrm{mg} / \mathrm{kg}(50 \mathrm{mg})$ alternate days for 10 weeks to a total dose of $1.75 \mathrm{~g}$ (started three weeks before the diagnosis of cerebral abscess); flucytosine $0.16 \mathrm{mg} / \mathrm{kg}(8 \mathrm{mg})$ daily for seven weeks; itraconazole $4 \mathrm{mg} / \mathrm{kg}(200 \mathrm{mg})$ daily for three weeks; benzylpenicillin was continued for a total of 12 weeks.

This treatment was reasonably well tolerated, although renal potassium and magnesium loss required considerable intravenous supplementation. Creatinine clearance remained normal. There was no evidence of additional myelotoxicity. A repeat abscess aspiration one month after starting triple antifungal treatment was sterile on culture.

The recovery period was complicated by life threatening pneumonitis. An open lung biopsy specimen showed fine nodular infiltrates suggestive of varicella zoster. Fungi were not detected. He improved on intravenous acyclovir $10 \mathrm{mg} / \mathrm{kg}$ daily. Amphotericin and flucytosine were stopped seven weeks after the latter was started because of the development of hepatitis that was believed to be drug induced. This resolved satisfactorily.

A repeat psychological examination, 12 weeks after the diagnosis of cerebral abscesses, showed nearly complete recovery of all neurological processes. A computed tomogram at this time still showed some lucency in the occipitoparietal lobes and in the right frontal lobe but without ring enhancement. The importance of these findings remains uncertain. He managed a successful return to school.

It had been intended to give intensified leukaemia treatment on day 29 , according to protocol, but the development of cerebral abscesses led to this being abandoned. Cranial irradiation, together with the intrathecal methotrexate due during it, was given, but was delayed. A year after diagnosis he had a marrow relapse on treatment and subsequently died. No postmortem examination was performed. 


\section{Discussion}

Fungal infection in neutropenic patients is common. In one postmortem study of young cancer patients, 23 out of 88 fatalities showed evidence of systemic fungal infection. ${ }^{1}$

Our patient was at particular risk of such an infection. The risk factors included prolonged neutropenia, prolonged steroid treatment, diabetes, and an indwelling Hickman catheter. ${ }^{2}$ In addition he was on prolonged broad spectrum antibiotics, the most important factor in the aetiology of serious and fatal candidiasis. The stopping of antibiotics in those who remain febrile and neutropenic, however, carries a very high risk of serious bacterial infections. ${ }^{3}$

Empirical antifungal treatment is advocated for neutropenic patients with an antibiotic resistant fever. ${ }^{145}$ Amphotericin is the only antifungal with proved efficacy in neutropenic patients and its use as a single agent in this situation is therefore recommended.$^{4}$ Indeed in one study nearly complete elimination of fungal infections was seen if amphotericin was started in those who remained febrile and neutropenic after a week of antibiotic treatment. ${ }^{1}$ Starting antifungals only late in antibiotic resistant neutropenic fever is known to result in an increased mortality from fungal infection, but the optimal time to start remains uncertain. ${ }^{15}$

In our patient amphotericin was started after six days of fever resistant to antibiotics. Despite this, symptomatic presentation of mycotic cerebral abscesses occurred 12 days later and viable Candida was isolated from these three weeks after the start of amphotericin treatment. Doubt about the efficacy of amphotericin alone as empirical treatment has been expressed previously. ${ }^{6}$

The use of flucytosine combined with amphoteri- cin has been suggested, not least because of its synergistic activity against Candida. ${ }^{6}$ This combination, however, has been considered to have a number of disadvantages-namely, the need to lower the dose of amphotericin, the problems of determining flucytosine concentrations, and myelotoxicity. ${ }^{5}$ While using this combination therapeutically in our patient none of these problems were seen. Amphotericin dosage was reasonable $(1 \mathrm{mg} / \mathrm{kg}$ on alternate days), flucytosine concentrations were readily determined by our laboratories, and myelotoxicity was not an important problem. The addition of itraconazole seemed justified by the extremely high mortality previously documented for this condition.

\footnotetext{
References

1 Pizzo PA, Robichaud KJ, Gill FA, Witebsky FG. Empiric antibiotic and antifungal therapy for cancer patients with prolonged fever and granulocytopenia. Am J Med 1982;72: 101-11.

${ }^{2}$ Warnock DW, Richardson MD. Fungal infections in the compromised patient. Chichester: Wiley, 1982.

${ }^{3}$ Pizzo PA, Robichaud KJ, Gill FA, et al. Duration of empiric antibody therapy in granulocytopenic patients with cancer. $A m J$ Med 1979;67:194-200.

4 Marcus RE, Goldman JM. Management of infection in the neutropenic patient. $\mathrm{Br}$ Med $J$ 1986;293:406-8.

5 Cohen J. Empirical antifungal therapy in neutropenic patients. Journal of Antimicrobal Chemotherapy 1984;13:409-11.

${ }^{6}$ Polak A. Combined therapy of systemic mycoses. Proceedings of 13th International Congress of Chemotherapy. Vienna: 1983. Clinical and experimental studies in immunotherapy. Amsterdam and Princeton: Excerpta Medica, 1984: Part 20:2-9.
}

Correspondence and requests for reprints to Dr MG Mott, Bristol Royal Hospital for Sick Children, St Michael's Hill, Bristol BS2 8BJ.

Accepted 29 December 1987
SUMMARY We recently reported an association between recurrent episodes of severe apnoea requiring vigorous resuscitation for which no cause could be found and episodic hypothermia. Two similar cases are now reported that give further evidence of a link between hypothermia and acute life threatening episodes of apnoea.
Our first report concerned a baby boy who at the time of writing this paper was 4 years old. ${ }^{1}$ He has had no further apnoeic attacks, but does have recurrent attacks of hypothermia (rectal temperature $32-35^{\circ} \mathrm{C}$ ). The only new development is that he now has occasional episodes of sweating and hypoglycaemia that have been documented while he was under observation in hospital. He is being monitored at home with a skin temperature probe. 